\title{
THE DIFFERENTIAL GEOMETRY OF ALMOST HERMITIAN ALMOST CONTACT METRIC SUBMERSIONS
}

\author{
T. TSHIKUNA-MATAMBA
}

Received 19 March 2003

\begin{abstract}
Three types of Riemannian submersions whose total space is an almost Hermitian almost contact metric manifold are studied. The study is focused on fundamental properties and the transference of structures.
\end{abstract}

2000 Mathematics Subject Classification: 53C15.

1. Introduction. In this paper, we discuss some geometric properties of Riemannian submersions whose total space is an almost Hermitian almost contact metric manifold.

If the base space is an almost quaternionic metric manifold, Watson has defined in $[12,13]$ a type of such submersions which we will call almost Hermitian almost contact metric submersion of type I. When the base space is an almost contact metric manifold with 3-structure, another type of these submersions called almost Hermitian almost contact metric submersions of type II has been introduced by the present author in [9]. Replacing the base space by an almost Hermitian almost contact metric manifold, we get a new type of such submersions, a third one, which we will call almost Hermitian almost contact metric submersions of type III. Note that this last type lies between almost Hermitian submersions studied by Watson [11] and almost contact metric submersions of type I $[6,8,15]$. Analogously, almost Hermitian almost contact metric submersions of type I lie between almost Hermitian submersions and almost contact metric submersions of type II $[6,8,15]$.

This text is organized in the following way.

Section 2 is devoted to the background of the manifolds which will be used in the sequel.

Section 3 is concerned with the properties of the three types of submersions under consideration. For each type, we have here examined:

(1) the structure of the base space and the fibres according to that of the total space;

(2) the classes of submersions with totally geodesic fibres;

(3) the classes of submersions preserving the holomorphic sectional curvature tensor of the vertical or of the horizontal vector fields.

In Section 4, we give some examples of these types of submersions.

Throughout this paper, arbitrary vector fields of the tangent space of a differentiable manifold $M$ will be denoted by $D, E$, and $G$.

2. Preliminaries on manifolds. Recall that an almost Hermitian manifold is a Riemannian manifold $(M, g)$ equipped with a tensor field $J$ of type $(1,1)$ such that the 
following two conditions are satisfied:

$\left(1^{\circ}\right)(J \circ J) E=-E$,

$\left(2^{\circ}\right) g(J D, J E)=g(D, E)$.

In the last case $\left(2^{\circ}\right), g$ is called a compatible metric and $(g, J)$ is an almost Hermitian structure on $M$. Any almost Hermitian manifold $(M, g, J)$ possesses a differential 2-form $\Omega$, called the fundamental 2-form or the Kähler 2-form, defined by $\Omega(D, E)=g(D, J E)$.

Denoting by $\nabla$ the Levi-Civita connection of $g$, and by $d \Omega$ the differential of $\Omega$, the following identities are well known:

$$
\begin{gathered}
\left(\nabla_{D} J\right) E=\nabla_{D}(J E)-J \nabla_{D} E, \\
\left(\nabla_{D} \Omega\right)(E, G)=g\left(E,\left(\nabla_{D} J\right) G\right)=-g\left(\left(\nabla_{D} J\right) E, G\right), \\
3 d \Omega(D, E, G)=\Im\left\{\left(\nabla_{D} \Omega\right)(E, G)\right\},
\end{gathered}
$$

where $\$$ denotes the cyclic sum on $D, E$, and $G$.

In [2], Gray and Hervella have obtained a classification of almost Hermitian structures. We recall the defining relations of those that will be needed later.

An almost Hermitian manifold $(M, g, J)$ is said to be

(a) Kählerian if $\nabla J=0$;

(b) nearly Kählerian if $\left(\nabla_{D} J\right) D=0$;

(c) $G_{1}$-manifold if $\left(\nabla_{D} \Omega\right)(D, E)-\left(\nabla_{J D} \Omega\right)(J D, E)=0$;

(d) $G_{2}$-manifold if $\Im\left\{\left(\nabla_{D} \Omega\right)(E, G)-\left(\nabla_{J D} \Omega\right)(J E, G)\right\}=0$.

The Nijenhuis tensor of $J$ will be denoted by $N$. An almost quaternionic metric manifold is a quintuple $\left(M, g, J_{1}, J_{2}, J_{3}\right)$, where

(a) $(M, g)$ is a Riemannian manifold;

(b) $\left(g, J_{i}\right)$ is an almost Hermitian structure on $M$ for $i=1,2,3$;

(c) $J_{i} \circ J_{j}=-J_{j} \circ J_{i}=J_{k}$, for $i<j, i \neq k$ and $j \neq k$.

For $i=1,2,3$, the analogues of identities (2.1), (2.2), and (2.3) are obtained as in the case of almost Hermitian manifolds. Almost quaternionic metric manifolds are of dimension $4 m$ and their nomenclature is related to that of almost Hermitian structures.

By an almost contact metric manifold, one understands a quintuple $(M, g, \varphi, \xi, \eta)$, where

$\left(1^{\circ}\right) \xi$ is a characteristic vector field;

$\left(2^{\circ}\right) \eta$ is a differential 1 -form such that $\eta(\xi)=1$;

$\left(3^{\circ}\right) \varphi$ is a tensor field of type $(1,1)$ satisfying $\varphi^{2} D=-D+\eta(D) \xi$;

$\left(4^{\circ}\right) \mathcal{g}(\varphi D, \varphi E)=g(D, E)-\eta(D) \eta(E)$.

In the last case, $g$ is called a compatible metric and $(g, \varphi, \xi, \eta)$ is an almost contact metric structure. Replacing $J$ by $\varphi$, the fundamental 2-form $\phi$ is defined by $\phi(D, E)=$ $g(D, \varphi E)$ and the analogues of identities (2.1), (2.2), and (2.3) hold. The covariant derivative of $\eta$ and the exterior differential of $\eta$ are defined, respectively, by

$$
\begin{gathered}
\left(\nabla_{D} \eta\right) E=g\left(E, \nabla_{D} \xi\right), \\
2 d \eta(D, E)=\left(\nabla_{D} \eta\right) E-\left(\nabla_{E} \eta\right) D .
\end{gathered}
$$


In [5], Sasaki and Hatakeyama have defined a tensor field $N^{(1)}$ of type $(1,2)$ by setting

$$
N^{(1)}(D, E)=[\varphi, \varphi](D, E)+2 d \eta(D, E) \xi
$$

where $[\varphi, \varphi]$ is the Nijenhuis tensor of $\varphi$.

Almost contact metric manifolds are of dimension $2 m+1$. We recall the defining relations of those which will be used in this study. An almost contact metric manifold $(M, g, \varphi, \xi, \eta)$ is called

(a) Sasakian if $\phi=d \eta$ and $N^{(1)}=0$;

(b) $G_{1}$-Sasakian if $\left(\nabla_{D} \varphi\right) D-\left(\nabla_{\varphi D} \varphi\right) \varphi D+\eta(D)\left(\nabla_{\varphi D} \xi\right)=0$;

(c) $G_{2}$-Sasakian if $\Im\left\{\left(\nabla_{D} \phi\right)(E, G)-\left(\nabla_{\varphi D} \phi\right)(\varphi E, G)-\eta(E)\left(\nabla_{\varphi D} \eta\right) G\right\}=0$;

(d) quasi-Sasakian if $d \phi=0=N^{(1)}$;

(e) cosymplectic if $\nabla \varphi=0$;

(f) closely cosymplectic if $\left(\nabla_{D} \varphi\right) D=0=d \eta$;

(g) nearly cosymplectic if $\left(\nabla_{D} \varphi\right) D=0$;

(h) nearly-K-cosymplectic if $\left(\nabla_{D} \varphi\right) E+\left(\nabla_{E} \varphi\right) D=0=\nabla_{D} \xi$.

Let $\left(\varphi_{i}, \xi_{i}, \eta_{i}\right)_{i=1}^{3}$ be three almost contact structures such that each of them is compatible with the Riemannian structure $g$. We say that $\left(M, g,\left(\varphi_{i}, \xi_{i}, \eta_{i}\right)_{i=1}^{3}\right)$ is an almost contact metric manifold with 3 -structure [3] if for any cyclic permutation $(i, j, k)$ of $\{1,2,3\}$ the following conditions are satisfied:

$\left(1^{\circ}\right) \eta_{i}\left(\xi_{j}\right)=\eta_{j}\left(\xi_{i}\right)=0$

$\left(2^{\circ}\right) \varphi_{i} \xi_{j}=-\varphi_{j} \xi_{i}=\xi_{k}$

$\left(3^{\circ}\right) \varphi_{i} \circ \varphi_{j}-\eta_{j} \otimes \xi_{i}=-\varphi_{j} \circ \varphi_{i}+\eta_{i} \otimes \xi_{j}=\varphi_{k}$;

$\left(4^{\circ}\right) \eta_{i} \circ \varphi_{j}=-\eta_{j} \circ \varphi_{i}=\eta_{k}$.

As in the case of almost quaternionic metric manifolds, the fundamental local 2-form $\phi_{i}$ is defined by $\phi_{i}(D, E)=g\left(D, \varphi_{i} E\right)$. Almost contact manifolds with 3-structure are of dimension $4 m+3$.

Let $(M, g, J)$ be an almost Hermitian manifold furnished with two almost contact structures $\left(\varphi_{i}, \xi_{i}, \eta_{i}\right)_{i=1}^{2}$. We say that $\left(M, g, J,\left(\varphi_{i}, \xi_{i}, \eta_{i}\right)_{i=1}^{2}\right)$ is an almost Hermitian almost contact metric manifold if

(a) each $\left(\varphi_{i}, \xi_{i}, \eta_{i}\right)$-structure is compatible with the metric $g$;

(b) $J \xi_{1}=-\xi_{2}, J \xi_{2}=\xi_{1}$;

(c) $\varphi_{i}^{2} D=-D+\eta_{1}(D) \xi_{1}+\eta_{2}(D) \xi_{2}$;

(d) $\varphi_{1}(J D)=-J\left(\varphi_{1} D\right)=\varphi_{2}(D)$;

(e) $\varphi_{1}\left(\varphi_{2} D\right)=-\varphi_{2}\left(\varphi_{1} D\right)=J D+\eta_{1}(D) \xi_{2}+\eta_{2}(D) \xi_{1}$;

(f) $\varphi_{2}(J D)=-J\left(\varphi_{2} D\right)=-\varphi_{1} D$.

Concerning the fundamental local 2-form, we have

$\left(1^{\circ}\right) \phi_{i}(D, E)=g\left(D, \varphi_{i} E\right)$ for $i=1,2$;

$\left(2^{\circ}\right) \Omega(D, E)=g(D, J E)$.

The dimension of this type of manifold is $4 m+2$. For the nomenclature, we will indicate the name of the almost contact metric structure followed by the name of the almost Hermitian one. For instance, a Sasakian-Kählerian manifold is a Kählerian manifold equipped with two Sasakian structures. 
3. The three types of almost Hermitian almost contact metric submersions. By a Riemannian submersion, one understands a submersion $f: M \rightarrow M^{\prime}$ between Riemannian manifolds such that $\left.f_{*}\right|_{\left(\operatorname{Ker} f_{*}\right) \perp}$ is a linear isometry [4]. The tangent bundle $T(M)$ of the total space $M$ admits an orthogonal decomposition $T(M)=V(M) \oplus H(M)$, where $V(M)$ and $H(M)$ denote, respectively, the vertical and the horizontal distributions. We denote by $\mathscr{V}$ and $\mathscr{H}$ the vertical and the horizontal projections of $T(M)$ onto $V(M)$ and $H(M)$, respectively. For all points $x^{\prime} \in M^{\prime}$, the closed, embedded submanifold $F_{x^{\prime}}=f^{-1}\left(x^{\prime}\right)$ is called the fibre of $f$ over $x^{\prime}$. It is known that $\operatorname{dim} F_{X^{\prime}}=\operatorname{dim} M-\operatorname{dim} M^{\prime}$.

A vector field $X$ of the horizontal distribution is called a basic vector field if it is $f$-related to a vector field $X_{*}$ of the base space $M^{\prime}$, that is, $X_{*}=f_{*} X$.

We will denote the horizontal vector fields by $X, Y$, and $Z$, while those of the vertical distribution will be denoted by $U, V$, and $W$. On the base space, tensors and other operators will be specified by a prime $\left({ }^{\prime}\right)$, while those of the fibres will be denoted by a caret $\left(^{\wedge}\right)$. For instance $\nabla, \nabla^{\prime}$, and $\hat{\nabla}$ will designate the Levi-Civita connection of the total space, the base, and the fibres, respectively.

3.1. Submersions of type I. Almost Hermitian almost contact metric submersions of type I were originally defined by Watson [12, 13].

DEFINITION 3.1. Let $\left(M^{4 m+2}, g, J,\left(\varphi_{i}, \xi_{i}, \eta_{i}\right)_{i=1}^{2}\right)$ be an almost Hermitian almost contact metric manifold and $\left(M^{\prime} 4 m^{\prime}, g^{\prime},\left(J_{i}^{\prime}\right)_{i=1}^{3}\right)$ an almost quaternionic metric manifold. A Riemannian submersion $f: M^{4 m+2} \rightarrow M^{\prime 4 m^{\prime}}$ is called an almost Hermitian almost contact metric submersion of type $I$ if

(a) $f_{*} J D=J_{1}^{\prime} f_{*} D$;

(b) $f_{*} \varphi_{1} D=J_{2}^{\prime} f_{*} D$;

(c) $f_{*} \varphi_{2} D=J_{3}^{\prime} f_{*} D$.

Proposition 3.2. Let $f:\left(M^{4 m+2}, g, J,\left(\varphi_{i}, \xi_{i}, \eta_{i}\right)_{i=1}^{2}\right) \rightarrow\left(M^{\prime 4 m^{\prime}}, g^{\prime},\left(J_{i}^{\prime}\right)_{i=1}^{3}\right)$ be an almost Hermitian almost contact metric submersion of type I. Then

(a) the vertical and horizontal distributions induced by $f$ are invariant by $J$ and by $\varphi_{i}$ for $i=1,2$;

(b) the vector fields $\xi_{1}$ and $\xi_{2}$ are vertical;

(c) $\eta_{i}(X)=0$ for $i=1,2$;

(d) $f^{*} \Omega_{1}^{\prime}=\Omega, f^{*} \Omega_{2}^{\prime}=\phi_{1}$, and $f^{*} \Omega_{3}^{\prime}=\phi_{2}$;

(e) $f_{*} N=N_{1}^{\prime}, f_{*} N_{1}^{(1)}=N_{2}^{\prime}$, and $f_{*} N_{2}^{(1)}=N_{3}^{\prime}$;

(f) $\hat{N}(U, V)=N(U, V), \hat{N}_{1}^{(1)}(U, V)=N_{1}^{(1)}(U, V)$, and $\hat{N}_{2}^{(1)}(U, V)=N_{2}^{(1)}(U, V)$.

Proof. Assertions (a), (b), and (c) are established as in [14]. We consider the case of $f^{*} \Omega_{1}^{\prime}=\Omega$. Let $X$ and $Y$ be two basic vector fields. We have

$$
\begin{aligned}
f^{*} \Omega_{1}^{\prime}(X, Y) & =f^{*} g^{\prime}\left(X_{*}, J_{1}^{\prime} Y_{*}\right) \\
& =g^{\prime}\left(f_{*} X, J_{1}^{\prime} f_{*} Y\right) \\
& =g^{\prime}\left(f_{*} X, f_{*} J Y\right) \\
& =f^{*} g^{\prime}(X, J Y)=g(X, J Y)=\Omega(X, Y)
\end{aligned}
$$

which gives the proof of assertion (d). 
Consider (e). Clearly we have

$$
f_{*} N(X, Y)=f_{*}[J, J](X, Y)=\left[J_{1}^{\prime}, J_{1}^{\prime}\right]\left(f_{*} X, f_{*} Y\right)=N^{\prime}\left(X_{*}, Y_{*}\right) .
$$

Other cases of this assertion can be established in an analogous manner.

Consider the last case, (f), and suppose that $U$ and $V$ are vector fields tangent to the fibres. By (2.6) we have $\hat{N}_{2}^{(1)}(U, V)=\left[\hat{\varphi}_{2}, \hat{\varphi}_{2}\right](U, V)+2 d \hat{\eta}_{2}(U, V) \hat{\xi}_{2}$. Since $\hat{\xi}_{2}=\xi_{2}$ and $d \hat{\eta}_{2}(U, V)=d \eta_{2}(U, V)$, the proof follows.

Proposition 3.3. Let $f:\left(M^{4 m+2}, g, J,\left(\varphi_{i}, \xi_{i}, \eta_{i}\right)_{i=1}^{2}\right) \rightarrow\left(M^{\prime 4 m^{\prime}}, g^{\prime},\left(J_{i}^{\prime}\right)_{i=1}^{3}\right)$ be an almost Hermitian almost contact metric submersion of type I. Then the fibres are almost Hermitian almost contact metric manifolds.

Proof. It is clear that the dimension of the fibres is $4\left(m-m^{\prime}\right)+2$. By the preceding Proposition 3.2, we have shown that the vertical distribution is invariant by $J, \varphi_{1}$, and $\varphi_{2}$. Moreover, $\xi_{1}$ and $\xi_{2}$ are vertical vector fields; so $\eta_{1}$ and $\eta_{2}$ do not vanish on the vertical distribution. Therefore, $\left(\hat{\varphi}_{1}, \hat{\xi}_{1}, \hat{\eta}_{1}\right)$ and $\left(\hat{\varphi}_{2}, \hat{\xi}_{2}, \hat{\eta}_{2}\right)$ are almost contact structures compatible with the metric tensor $\hat{g}$ on the fibres. It is not hard to show that $(\hat{\mathfrak{g}}, \hat{J})$ is an almost Hermitian structure so that $\left(F^{4\left(m-m^{\prime}\right)+2}, \hat{g}, \hat{J},\left(\hat{\varphi}_{i}, \hat{\xi}_{i}, \hat{\eta}_{i}\right)_{i=1}^{2}\right)$ is an almost Hermitian almost contact metric manifold.

Proposition 3.4. Let $f:\left(M^{4 m+2}, g, J,\left(\varphi_{i}, \xi_{i}, \eta_{i}\right)_{i=1}^{2}\right) \rightarrow\left(M^{\prime 4 m^{\prime}}, g^{\prime},\left(J_{i}^{\prime}\right)_{i=1}^{3}\right)$ be an almost Hermitian almost contact metric submersion of type I. If the total space is SasakianKählerian, then

(a) the base space is quaternionic Kählerian;

(b) the fibres are Sasakian-Kählerian.

Proof. (a) By virtue of the defining relations of a Sasakian-Kählerian structure, $\phi_{1}=$ $d \eta_{1}, \phi_{2}=d \eta_{2}$, and $N=N_{1}^{(1)}=N_{2}^{(1)}=0=d \Omega$ so that $d \phi_{1}=d \phi_{2}=0$ from which we deduce $d \Omega_{2}^{\prime}=d \Omega_{3}^{\prime}$ and $N_{1}^{\prime}=0=N_{2}^{\prime}=N_{3}^{\prime}=d \Omega_{1}^{\prime}$ by Proposition 3.2. It is clear that the base space is defined by $d \Omega_{i}^{\prime}=0=N_{i}^{\prime}$ which are the defining relations of a Kählerian structure.

(b) By Proposition 3.2, it is shown that $\hat{N}(U, V)=0=\hat{N}_{1}^{(1)}(U, V)$. Since the fibres are invariant by $\varphi_{i}$ and $J$, then $\phi_{1}=d \eta_{1}, \phi_{2}=d \eta_{2}$, and $d \Omega=0$. Therefore, the fibres have two Sasakian structures and a Kählerian one.

On the total space of a Riemannian submersion, O’Neill [4] defined two tensor fields $T$ and $A$ of type $(1,2)$ by setting

$$
\begin{aligned}
& T_{D} E=\mathscr{H} \nabla_{\mathscr{V} D} \mathscr{V} E+\mathscr{V} \nabla_{\mathscr{V} D} \mathscr{H} E, \\
& A_{D} E=\mathscr{V} \nabla_{\mathscr{H} D} \mathscr{H} E+\mathscr{H} \nabla_{\mathscr{H} D} \mathscr{V} E .
\end{aligned}
$$

It is known that the configuration tensor $A$ measures the integrability of the horizontal distribution, while the configuration tensor $T$ is a tool in the study of the geometry of the fibres being essentially the second fundamental form of the embedding of the fibre submanifolds into the total space. 
LEMMA 3.5. Let $f:\left(M^{4 m+2}, g, J,\left(\varphi_{i}, \xi_{i}, \eta_{i}\right)_{i=1}^{2}\right) \rightarrow\left(M^{\prime 4 m^{\prime}}, g^{\prime},\left(J_{i}^{\prime}\right)_{i=1}^{3}\right)$ be an almost Hermitian almost contact metric submersion of type I. If, for $i=1,2, T_{U} V+T_{\varphi_{i} U}\left(\varphi_{i} V\right)=$ 0 and $T_{U} V+T_{J U} J V=0$, then $T=0$.

Proof. Since $0=T_{U} V+T_{\varphi_{1} U}\left(\varphi_{1} V\right)=T_{U} V+T_{\varphi_{2} U}\left(\varphi_{2} V\right)=T_{U} V+T_{J U} J V$, we have $T_{\varphi_{1} U}\left(\varphi_{1} V\right)=T_{\varphi_{2} U}\left(\varphi_{2} V\right)=T_{J U} J V$. Therefore, $-T_{U} V+\eta_{1}\left(T_{U} V\right) \xi_{1}=-T_{U} V$ and $-T_{U} V+$ $\eta_{2}\left(T_{U} V\right) \xi_{2}=-T_{U} V$ which implies that $\eta_{1}\left(T_{U} V\right) \xi_{1}=\eta_{2}\left(T_{U} V\right) \xi_{2}$ and shows that $T_{U} V=$ 0 . Then, we get $T=0$.

Corollary 3.6. Let $f:\left(M^{4 m+2}, g, J,\left(\varphi_{i}, \xi_{i}, \eta_{i}\right)_{i=1}^{2}\right) \rightarrow\left(M^{\prime 4 m^{\prime}}, g^{\prime},\left(J_{i}^{\prime}\right)_{i=1}^{3}\right)$ be an almost Hermitian almost contact metric submersion of type I. If for $i=1,2, T_{U}\left(\varphi_{i} V\right)=$ $\varphi_{i} T_{U} V$ and $T_{U} J V=J T_{U} V$, then $T=0$.

Proof. If $T_{U}\left(\varphi_{i} V\right)=\varphi_{i} T_{U} V$, then $T_{\varphi_{i} U}\left(\varphi_{i} V\right)=\varphi_{i} T_{\varphi_{i} U} V$; since $\varphi_{i} U$ is vertical and $T$ is symmetric on vertical vector fields, then $T_{\varphi_{i} U} V=T_{V}\left(\varphi_{i} U\right)=\varphi_{i} T_{V} U=\varphi_{i} T_{U} V$. It is clear that $T_{\varphi_{i} U}\left(\varphi_{i} V\right)=\varphi_{i}^{2} T_{U} V=-T_{U} V+\eta_{i}\left(T_{U} V\right) \xi_{i}$. On the other hand, we can show that, in this case, $T_{U} \xi_{i}=0$ which implies that $\eta_{i}\left(T_{U} V\right)=0$; we then obtain $T_{\varphi_{i} U}\left(\varphi_{i} V\right)=$ $-T_{U} V$ from which we deduce that $T_{U} V+T_{\varphi_{i} U}\left(\varphi_{i} V\right)=0$. Applying the lemma, we get $T=0$.

THEOREM 3.7. Let $f:\left(M^{4 m+2}, g, J,\left(\varphi_{i}, \xi_{i}, \eta_{i}\right)_{i=1}^{2}\right) \rightarrow\left(M^{\prime 4 m^{\prime}}, g^{\prime},\left(J_{i}^{\prime}\right)_{i=1}^{3}\right)$ be an almost Hermitian almost contact metric submersion of type I. If the total space is SasakianKählerian, closely cosymplectic nearly Kählerian, nearly K-cosymplectic nearly Kählerian, or nearly cosymplectic nearly Kählerian, then the fibres are totally geodesic.

Proof. We recall that the fibres of a Riemannian submersion are totally geodesic if the configuration tensor $T$ vanishes identically. For each of the manifolds in hand, it is not hard to show that the preceding corollary applies.

In [4, page 465], O’Neill has shown that a Riemannian submersion is Riemannian sectional curvature increasing on horizontal tangent planes. In the following, we will investigate the relations between the holomorphic sectional curvature tensors of the total space, of the base space, and of the fibres. We will be interested in the case where equality can be obtained. In other words, we want to determine the classes of almost Hermitian almost contact metric submersions which preserve the holomorphic sectional curvature tensor on the horizontal and eventually on the vertical vector fields.

Recall that for an almost contact metric manifold $(M, g, \varphi, \xi, \eta)$, the $\varphi$-holomorphic sectional curvature tensor is defined by

$$
H_{\varphi}(E)=\|E\|^{-4} g(R(E, \varphi E) E, \varphi E)
$$

where $g(E, \xi)=0$.

Proposition 3.8. Let $f:\left(M^{4 m+2}, g, J,\left(\varphi_{i}, \xi_{i}, \eta_{i}\right)_{i=1}^{2}\right) \rightarrow\left(M^{\prime 4 m^{\prime}}, g^{\prime},\left(J_{i}^{\prime}\right)_{i=1}^{3}\right)$ be an almost Hermitian almost contact metric submersion of type I. Then, the holomorphic sectional curvature tensors satisfy 
(a) $H \varphi_{i}(U)=\hat{H} \varphi_{i}(U)+\|U\|^{-4}\left\{\left\|T_{U}\left(\varphi_{i} U\right)\right\|^{2}-g\left(T_{U} U, T_{\varphi_{i} U}\left(\varphi_{i} U\right)\right)\right\}$ for $i=1,2$,

(b) $H_{J}(U)=H_{\hat{J}}(U)+\|U\|^{-4}\left\{\left\|T_{U} J U\right\|^{2}-g\left(T_{U} U, T_{J U} J U\right)\right\}$,

(c) $H_{J}(X)=H_{J_{1}^{\prime}}\left(X_{*}\right)-3\|X\|^{-4}\left\|A_{X} J X\right\|^{2}$,

(d) $H_{\varphi_{1}}(X)=H_{J_{2}^{\prime}}\left(X_{*}\right)-3\|X\|^{-4}\left\|A_{X}\left(\varphi_{1} X\right)\right\|^{2}$,

(e) $H \varphi_{2}(X)=H_{J_{3}^{\prime}}\left(X_{*}\right)-3\|X\|^{-4}\left\|A_{X}\left(\varphi_{2} X\right)\right\|^{2}$.

Proof. It is an adaptation of [14, Theorem 5.1].

Proposition 3.9. Let $f:\left(M^{4 m+2}, g, J,\left(\varphi_{i}, \xi_{i}, \eta_{i}\right)_{i=1}^{2}\right) \rightarrow\left(M^{\prime 4 m^{\prime}}, g^{\prime},\left(J_{i}^{\prime}\right)_{i=1}^{3}\right)$ be an almost Hermitian almost contact metric submersion of type I. Let $X$ be a basic vector field on M. If the total space is Sasakian-Kählerian, quasi-Sasakian-Kählerian, cosymplecticKählerian, or closely cosymplectic nearly Kählerian, then

(a) $H_{\varphi_{i}}(U)=\hat{H}_{\hat{\varphi}_{i}}(U)$, for $i=1,2$,

(b) $H_{J}(U)=H_{\hat{J}}(U)$,

(c) $H_{\varphi_{1}}(X)=H_{J_{2}^{\prime}}\left(X_{*}\right)$,

(d) $H_{\varphi_{2}}(X)=H_{J_{3}^{\prime}}\left(X_{*}\right)$

(e) $H_{J}(X)=H_{J_{1}^{\prime}}\left(X_{*}\right)$.

Proof. Since $T=0$ by Theorem 3.7, we get the proofs of (a) and (b). Assertions (c), (d), and (e) follow from the fact that $A_{J X} Y=0=J A_{X} Y, A_{\varphi_{1} X} Y=\varphi_{1} A_{X} Y$, and $A_{\varphi_{2} X} Y=\varphi_{2} A_{X} Y$ from which we deduce $A_{X} J X=0=A_{X}\left(\varphi_{1} X\right)=A_{X}\left(\varphi_{2} X\right)$.

Now, we want to examine the properties of a type of submersions which is closely related to those initiated by Chinea [1].

\subsection{Submersions of type II}

DEFINITION 3.10. Let $\left(M^{4 m+2}, \mathfrak{g}, J,\left(\varphi_{i}, \xi_{i}, \eta_{i}\right)_{i=1}^{2}\right)$ be an almost Hermitian almost contact metric manifold and let $\left(M^{\prime 4 m^{\prime}+3}, g^{\prime},\left(\varphi_{i}^{\prime}, \xi_{i}^{\prime}, \eta_{i}^{\prime}\right)_{i=1}^{3}\right)$ be an almost contact metric manifold with 3-structure. We say that a Riemannian submersion $f: M^{4 m+2} \rightarrow M^{\prime 4 m^{\prime}+3}$ is an almost Hermitian almost contact metric submersion of type II if it satisfies

(a) $f_{*}\left(\varphi_{i} E\right)=\varphi_{i}^{\prime} f_{*} E$, for $i=1,2$,

(b) $f_{*} J E=\varphi_{3}^{\prime} f_{*} E-\eta_{3}^{\prime}\left(f_{*} E\right) \xi_{3}^{\prime}$,

(c) $f_{*} \xi_{1}=\xi_{1}^{\prime}$ and $f_{*} \xi_{2}=\xi_{2}^{\prime}$.

Proposition 3.11. Let $f:\left(M^{4 m+2}, g, J,\left(\varphi_{i}, \xi_{i}, \eta_{i}\right)_{i=1}^{2}\right) \rightarrow\left(M^{\prime 4 m^{\prime}+3}, g^{\prime},\left(\varphi_{i}^{\prime}, \xi_{i}^{\prime}, \eta_{i}^{\prime}\right)_{i=1}^{3}\right)$ be an almost Hermitian almost contact metric submersion of type II. Then,

(a) the vertical and horizontal distributions induced by $f$ are invariant by $\varphi_{1}$ and $\varphi_{2}$;

(b) the vector fields $\xi_{1}$ and $\xi_{2}$ are horizontal;

(c) $\eta_{i}(U)=0$ for $i=1,2$;

(d) the fibres are almost contact metric manifolds, invariant by $\varphi_{i}$ for $i=1,2$, and semi-invariant by $\mathrm{J}$;

(e) $\hat{\eta}(J V)=0$ for all $V$ tangent to the fibres.

Proof. See $[9,10]$. 
Let $\mu$ be a unitary vector field of $M$ which is orthogonal to the fibres. We recall from [9] that the almost contact structure $(\hat{\varphi}, \hat{\xi}, \hat{\eta})$ on the fibres is given by

$$
\begin{gathered}
\hat{\xi}=-J \mu, \\
\hat{\eta}(E)=g(J E, \mu), \\
\hat{\varphi}(E)=J E-\hat{\eta}(E) \mu .
\end{gathered}
$$

THEOREM 3.12. Let $f:\left(M^{4 m+2}, g, J,\left(\varphi_{i}, \xi_{i}, \eta_{i}\right)_{i=1}^{2}\right) \rightarrow\left(M^{\prime 4 m^{\prime}+3}, g^{\prime},\left(\varphi_{i}^{\prime}, \xi_{i}^{\prime}, \eta_{i}^{\prime}\right)_{i=1}^{3}\right)$ be an almost Hermitian almost contact metric submersion of type II with $\mu$ analytic and $J \mu$ parallel. If the total space is $G_{1}$ - (resp., $G_{2}$-) Sasakian-Kählerian, then the base space is a $\left(3-G_{1}\right)$ - (resp., $\left(3-G_{2}\right)$-) Sasakian manifold, while the fibres are $G_{1}$ - (resp., $G_{2}$-) Sasakian.

Proof. We examine the structure of the base space when the total space is a $G_{1^{-}}$Sasakian-Kählerian manifold. Consider a basic vector field $X$. We have to show that the base space is defined by $\left(\nabla_{X_{*}}^{\prime} \varphi_{i}^{\prime}\right) X_{*}-\left(\nabla^{\prime} \varphi_{i X_{*}}^{\prime} \varphi_{i}^{\prime}\right)\left(\varphi_{i}^{\prime} X_{*}\right)+\eta_{i}^{\prime}\left(X_{*}\right)\left(\nabla^{\prime} \varphi_{i X_{*}}^{\prime} \xi_{i}\right)=0$, where $i=1,2,3$. Since the total space is a $G_{1}$-Sasakian-Kählerian manifold, we have

$$
\begin{gathered}
\left(\nabla_{X} \varphi_{i}\right) X-\left(\nabla_{\varphi_{i} X}\left(\varphi_{i}\right)\right)\left(\varphi_{i} X\right)+\eta_{i}(X)\left(\nabla_{\varphi_{i} X} \xi_{i}\right)=0 \quad \text { for } i=1,2, \\
\left(\nabla_{X} J\right) X=0,
\end{gathered}
$$

which leads to

$$
\begin{gathered}
\left(\nabla_{X} \varphi_{i}\right) X-\varphi_{i} \nabla_{X} X+\left(\nabla_{\varphi_{i} X}\right) X+\varphi_{i} \nabla_{\varphi_{i} X}\left(\varphi_{i} X\right)=0, \\
\nabla_{X} J X-J \nabla_{X} X=0 .
\end{gathered}
$$

Using the fact that $f_{*}(J X)=\varphi_{3}^{\prime} f_{*} X-\eta_{3}^{\prime}\left(f_{*} X\right) \xi_{3}^{\prime}$, a straightforward computation of $f_{*}\left(\nabla_{X}\left(\varphi_{i} X\right)-\varphi_{i} \nabla_{X} X+\nabla_{\varphi_{i} X} X+\varphi_{i} \nabla_{\varphi_{i} X}\left(\varphi_{i} X\right)\right)$ and $f_{*}\left(\nabla_{X} J X-J \nabla_{X} X\right)$ establishes this part, keeping in mind that $\mu$ is analytic and $J \mu$ is parallel. The case where the total space is $G_{2}$-Sasakian-Kählerian is treated in the same way. Now, we examine the structure of the fibres when the total space is a $G_{2}$-Sasakian-Kählerian manifold. We want to show that $\Im\left\{\left(\hat{\nabla}_{U} \hat{\phi}\right)(V, W)-\left(\hat{\nabla}_{\hat{\varphi} U} \hat{\phi}\right)(\hat{\varphi} V, W)-\hat{\eta}(V)\left(\hat{\nabla}_{\hat{\varphi} U} \hat{\eta}\right) W\right\}=0$, where $U$, $V$, and $W$ are vector fields tangent to the fibres. In [9], it is shown that $\hat{\phi}(U, V)=\Omega(U, V)$, from which we have

$$
\left(\hat{\nabla}_{U} \hat{\phi}\right)(V, W)=\left(\nabla_{U} \Omega\right)(V, W)
$$

The analyticity of $\mu$ gives

$$
\left(\hat{\nabla}_{\hat{\varphi} U} \hat{\phi}\right)(\hat{\varphi} V, W)=\left(\hat{\nabla}_{J U} \Omega\right)(J V, W) .
$$

On the other hand, $\left(\hat{\nabla}_{\hat{\varphi} U} \hat{\eta}\right) W=\left(\hat{\nabla}_{J U} \hat{\eta}\right) W-\hat{\eta}(U)\left(\hat{\nabla}_{\mu} \hat{\eta}\right) W$; since $\mu$ is analytic, we have $\left(\hat{\nabla}_{\mu} \hat{\eta}\right) W=0$ so that we get

$$
\left(\hat{\nabla}_{\hat{\varphi} U} \hat{\eta}\right) W=\left(\hat{\nabla}_{J U} \hat{\eta}\right) W .
$$


But, by virtue of (2.4), $\left(\hat{\nabla}_{J U} \hat{\eta}\right) W=g\left(W, \hat{\nabla}_{J U} \hat{\xi}\right)$ which leads to

$$
\left(\hat{\nabla}_{\hat{\varphi} U} \hat{\eta}\right) W=0
$$

because $\hat{\xi}=-J \mu$ and $J \mu$ is parallel. Relations (3.8), (3.9), and (3.11) then give

$$
\begin{gathered}
\Im\left\{\left(\hat{\nabla}_{U} \hat{\phi}\right)(V, W)-\left(\hat{\nabla}_{\hat{\varphi} U} \hat{\phi}\right)(\hat{\varphi} V, W)-\hat{\eta}(U)\left(\hat{\nabla}_{\hat{\varphi} U} \hat{\eta}\right) W\right\} \\
=\mathfrak{G}\left\{\left(\nabla_{U} \Omega\right)(V, W)-\left(\nabla_{J U} \Omega\right)(J V, W)\right\} .
\end{gathered}
$$

The right-hand side of (3.12) is the defining relation of a $G_{2}$-manifold; therefore it vanishes and the proof is obtained.

\subsection{Submersions of type III}

DEFINITION 3.13. Let $\left(M^{4 m+2}, g, J,\left(\varphi_{i}, \xi_{i}, \eta_{i}\right)_{i=1}^{2}\right)$ and $\left(M^{4 m^{\prime}+2}, g^{\prime}, J^{\prime},\left(\varphi_{i}^{\prime}, \xi_{i}^{\prime}, \eta_{i}^{\prime}\right)_{i=1}^{2}\right)$ be almost Hermitian almost contact metric manifolds. By an almost Hermitian almost contact metric submersion of type III we mean a Riemannian submersion

$$
f: M^{4 m+2} \longrightarrow M^{\prime 4 m^{\prime}+2}
$$

such that

(a) $f_{*} \varphi_{i} E=\varphi_{i}^{\prime} f_{*} E$, for $i=1,2$,

(b) $f_{*} J E=J^{\prime} f_{*} E$

(c) $f^{*} \xi_{i}=\xi_{i}$, for $i=1,2$.

Proposition 3.14. Let $f:\left(M^{4 m+2}, g, J,\left(\varphi_{i}, \xi_{i}, \eta_{i}\right)_{i=1}^{2}\right) \rightarrow\left(M^{4 m^{\prime}+2}, g^{\prime}, J^{\prime},\left(\varphi_{i}^{\prime}, \xi_{i}^{\prime}\right.\right.$, $\left.\eta_{i}^{\prime}\right)_{i=1}^{2}$ ) be an almost Hermitian almost contact metric submersion of type III. Then,

(a) $f^{*} \eta_{i}^{\prime}=\eta_{i}$, for $i=1,2$,

(b) $f^{*} \phi_{i}^{\prime}=\phi_{i}$, for $i=1,2$,

(c) $f^{*} \Omega^{\prime}=\Omega$,

(d) the vertical and horizontal distributions are invariant by $J$ and $\varphi_{i}$ for $i=1,2$,

(e) the fibres are almost quaternionic metric manifolds,

(f) $\xi_{i}$ is horizontal for $i=1,2$,

(g) if $V$ is vertical, then $\eta_{i}(V)=0$ for $i=1,2$.

Proof. Each of the proofs of (a), (b), (c), (d), (f), and (g) is an adaptation of what is done in $[6,15]$. We can examine the case of (e). It is clear that the dimension of the fibres is $4\left(m-m^{\prime}\right)$ which is a multiple of 4 . We define three almost Hermitian structures $\left(\hat{\mathfrak{g}}, \hat{J}_{i}\right)$, where $i=1,2,3$, by setting

$$
\begin{aligned}
& \hat{J}_{1} U=J U, \\
& \hat{J}_{2} U=\varphi_{1} U, \\
& \hat{J}_{3} U=\varphi_{2} U .
\end{aligned}
$$

Considering $\left(\hat{g}, \hat{J}_{2}\right)$, we have $\left(\hat{J}_{2}\right)^{2} U=\varphi_{1}\left(\varphi_{1} U\right)=-U+\eta_{1}(U) \xi_{1}=-U$ because $\eta_{1}(U)=$ 0 . On the other hand, $\hat{g}\left(\hat{J}_{2} U, \hat{J}_{2} V\right)=\hat{\mathfrak{g}}\left(\varphi_{1} U, \varphi_{1} V\right)=-\hat{\mathfrak{g}}\left(U,\left(\varphi_{1}\right)^{2} V\right)=-\hat{\mathfrak{g}}(U,-V)+$ $\eta_{1}(V) \xi_{1}$; since $\eta_{1}(V)=0$, then $\hat{\mathfrak{g}}\left(\hat{J}_{2} U, \hat{J}_{2} V\right)=\hat{\mathfrak{g}}(U, V)$. We have thus shown that $\left(\hat{\mathfrak{g}}, \hat{J}_{2}\right)$ 
is an almost Hermitian structure. By the same procedure, we can show that $\left(\hat{g}, \hat{J}_{1}\right)$ and $\left(\hat{\mathfrak{g}}, \hat{J}_{3}\right)$ are also almost Hermitian structures. It remains to show that $\hat{J}_{2} \circ \hat{J}_{3}=\hat{J}_{1}$. In fact, $\left(\hat{J}_{2} \circ \hat{J}_{3}\right)(U)=\hat{J}_{2}\left(\hat{J}_{3} U\right)=\hat{J}_{2}\left(\varphi_{2} U\right)=\varphi_{1}\left(\varphi_{2} U\right)$. Since $\left(\varphi_{1} \circ \varphi_{2}\right)(U)=J U+\eta_{1}(U) \xi_{2}+$ $\eta_{2}(U) \xi_{1}$ and $\eta_{i}(U)=0$, then $\left(\varphi_{1} \circ \varphi_{2}\right)(U)=J U=\hat{J}_{1} U$ which shows that $\hat{J}_{2} \circ \hat{J}_{3}=\hat{J}_{1}$. In the same manner, we can show that $\hat{J}_{3} \circ \hat{J}_{1}=\hat{J}_{2}=-\hat{J}_{1} \circ \hat{J}_{3}$ and $\hat{J}_{3} \circ \hat{J}_{2}=-\hat{J}_{1} U=-\hat{J}_{2} \circ \hat{J}_{3}$.

Proposition 3.15. Let $f:\left(M^{4 m+2}, g, J,\left(\varphi_{i}, \xi_{i}, \eta_{i}\right)_{i=1}^{2}\right) \rightarrow\left(M^{4 m^{\prime}+2}, g^{\prime}, J^{\prime},\left(\varphi_{i}^{\prime}, \xi_{i}^{\prime}\right.\right.$, $\left.\eta_{i}^{\prime}\right)_{i=1}^{2}$ ) be an almost Hermitian almost contact metric submersion of type III. Then,

(a) $\hat{\Omega}_{1}(U, V)=\Omega(U, V)$;

(b) $\hat{\Omega}_{2}(U, V)=\phi_{1}(U, V)$;

(c) $\hat{\Omega}_{3}(U, V)=\phi_{2}(U, V)$;

(d) $\mathscr{H}\left(\nabla_{X} \varphi_{i}\right) Y$ is the basic vector field associated to $\left(\nabla_{X_{*}}^{\prime} \varphi_{i}^{\prime}\right) Y_{*}$ and $\mathscr{H}\left(\nabla_{X} J\right) Y$ is the basic vector field associated to $\left(\nabla_{X_{*}}^{\prime} J^{\prime}\right) Y_{*}$, when $X$ and $Y$ are basic.

Proof. We refer to [15].

Concerning the structures of the base space and the fibres, one can see that this type of submersions is closely related to the almost contact metric submersions of type I [15]. Thus, we can easily obtain the properties similar to those established in [7, 8]. For instance, [7, Proposition 2.3] can be reformulated in the following way.

Proposition 3.16. Let $f:\left(M^{4 m+2}, g, J,\left(\varphi_{i}, \xi_{i}, \eta_{i}\right)_{i=1}^{2}\right) \rightarrow\left(M^{4 m^{\prime}+2}, g^{\prime}, J^{\prime},\left(\varphi_{i}^{\prime}, \xi_{i}^{\prime}\right.\right.$, $\left.\eta_{i}^{\prime}\right)_{i=1}^{2}$ ) be an almost Hermitian almost contact metric submersion of type III. If the total space is cosymplectic Kählerian, quasi-Sasakian-Kählerian, or Kenmotsu-Kählerian manifold, then the base space inherits the structure of the total space, while the fibres are almost quaternionic Kählerian.

Proof. It is clear that the manifolds under consideration have in common the following defining relations:

$$
d \phi_{i}(D, E, G)=\frac{b}{3} \lessgtr\left\{\eta_{i}(D) \phi_{i}(E, G)\right\}
$$

where $b$ is some real number;

$$
\begin{gathered}
N_{i}^{(1)}=0, \\
d \Omega(D, E, G)=0=N .
\end{gathered}
$$

In fact, if $b=0$, we obtain one of the defining relations of a cosymplectic Kählerian and quasi-Sasakian-Kählerian manifolds. Taking $b=2$, we get one of the defining relations of Kenmotsu-Kählerian manifolds. Concerning the structure of the base space, we have to show that

$$
\begin{gathered}
d \phi_{i}^{\prime}\left(X_{*}, Y_{*}, Z_{*}\right)=\frac{b}{3} \lessgtr\left\{\eta_{i}^{\prime}\left(X_{*}\right) \phi_{i}^{\prime}\left(Y_{*}, Z_{*}\right)\right\}, \\
N_{i}^{\prime(1)}=0, \\
d \Omega^{\prime}\left(X_{*}, Y_{*}, Z_{*}\right)=0=N^{\prime}
\end{gathered}
$$


and eventually,

$$
d \eta^{\prime}=0
$$

Since, by Proposition 3.14, we have $f^{*} \eta_{i}^{\prime}=\eta_{i}$ and $f^{*} \phi_{i}^{\prime}=\phi_{i}$, then $f^{*} d \eta_{i}^{\prime}=d \eta_{i}$ and $f^{*} d \phi_{i}^{\prime}=d \phi_{i}$, respectively; hence $d \phi_{i}^{\prime}\left(X_{*}, Y_{*}, Z_{*}\right)=(b / 3) \lessgtr\left\{\eta_{i}^{\prime}\left(X_{*}\right) \phi_{i}^{\prime}\left(Y_{*}, Z_{*}\right)\right\}$. From Proposition 3.14(c), we deduce that $d \Omega^{\prime}=0$ because $d \Omega=0$. As $d \eta_{i}=0$ and $f^{*} d \eta_{i}^{\prime}=$ $d \eta_{i}$, then $d \eta_{i}^{\prime}=0$ because $f^{*}$ is an isometry. It is not hard to show that $f_{*} N_{i}^{(1)}=N_{i}^{\prime(1)}$ and $f_{*} N=N^{\prime}$, from which we get $N_{i}^{\prime(1)}=0=N^{\prime}$. Concerning the structure of the fibres, we have to show that $d \hat{\Omega}_{1}=d \hat{\Omega}_{2}=d \hat{\Omega}_{3}=0$ and $\hat{N}_{1}^{(1)}=\hat{N}_{2}^{(1)}=0=N$. Since by Proposition 3.15(a), $\hat{\Omega}_{1}(U, V)=\hat{\Omega}(U, V)$, then $d \hat{\Omega}_{1}=d \Omega$ from which $d \hat{\Omega}_{1}=0$ because $d \Omega=0$. Again, by Proposition 3.15(b) and (c), we have $\phi_{1}(U, V)=\hat{\Omega}_{2}(U, V)$ and $\phi_{2}(U, V)=\hat{\Omega}_{3}(U, V)$. On the other hand, the vanishing of $\eta_{i}$ on vertical vector fields leads to $d \phi_{i}(U, V, W)=0$, from which $d \hat{\Omega}_{2}(U, V, W)=0=d \hat{\Omega}_{3}(U, V, W)$ follows. Since $N_{i}^{(1)}=0=N$, we have $\hat{N}_{i}^{(1)}=0=N$ which achieves the proof.

We omit the properties of holomorphic sectional curvature tensor because they are the same as in the case of almost contact metric submersions of type I [15].

\section{Some examples}

4.1. Submersions of type I. It is known that the quaternionic projective space $P_{m}(\mathbb{H})$ is an almost quaternionic metric manifold. In [12], Watson has shown that the canonical mapping

$$
P_{1}(\mathbb{C}) \longrightarrow P_{2 m+1}(\mathbb{C}) \stackrel{f}{\longrightarrow} P_{m}(\mathbb{Q})
$$

is an almost Hermitian almost contact metric submersion of type I.

Let $\bar{M}=M^{\prime 4 m^{\prime}} \times M^{4 m+2}$ be the product of an almost quaternionic metric manifold by an almost Hermitian almost contact metric one. It is clear that the manifold product $\bar{M}$ is of dimension $4\left(m^{\prime}+m\right)+2$ which we denote by $4 n+2$. We define an almost Hermitian almost contact metric structure $\left(\bar{g}, \bar{J},\left(\bar{\varphi}_{i}, \bar{\xi}_{i}, \bar{\eta}_{i}\right), i=1,2\right)$ by setting

$$
\begin{gathered}
\bar{\varphi}_{1}\left(D^{\prime}, D\right)=\left(J_{2}^{\prime} D^{\prime}, \varphi_{1} D\right) ; \\
\bar{\varphi}_{2}\left(D^{\prime}, D\right)=\left(J_{3}^{\prime} D^{\prime}, \varphi_{2} D\right) ; \\
\bar{J}\left(D^{\prime}, D\right)=\left(J_{1}^{\prime} D^{\prime}, J D\right) ; \\
\bar{\xi}_{i}=\frac{n}{m}\left(0, \xi_{i}\right), \quad \text { for } i=1,2 ; \\
\bar{\eta}_{i}\left(D^{\prime}, D\right)=\frac{m}{n} \eta_{i}(D), \quad \text { for } i=1,2 ; \\
\bar{g}\left(\left(D^{\prime}, D\right),\left(E^{\prime}, E\right)\right)=g^{\prime}\left(D^{\prime}, E^{\prime}\right)+\frac{n^{2}}{m^{2}} g(D, E),
\end{gathered}
$$

where $D^{\prime} \in T\left(M^{\prime}\right)$ and $D \in T(M)$. 
So, the projection $f: M^{\prime 4 m^{\prime}} \times M^{4 m+2} \rightarrow M^{\prime 4 m^{\prime}}$ is an almost Hermitian almost contact metric submersion of type I. Indeed, we have $f_{*} \bar{J}\left(D^{\prime}, D\right)=f_{*}\left(J_{1}^{\prime} D^{\prime}, J D\right)=J_{1}^{\prime} D^{\prime}$; since $D^{\prime}=f_{*}\left(D^{\prime}, D\right)$, we see that $f_{*} \bar{J}\left(D^{\prime}, D\right)=J_{1}^{\prime} f_{*}\left(D^{\prime}, D\right)$ which shows that $f_{*} \bar{J}=J_{1}^{\prime} f_{*}$. Similarly, $f_{*} \bar{\varphi}_{1}=J_{2}^{\prime} f_{*}$ and $f_{*} \bar{\varphi}_{2}=J_{3}^{\prime} f_{*}$. This example is suggested by one that was given in [6].

4.2. Submersions of type II. Following Watson [14, page 680], we can construct an example of almost Hermitian almost contact metric submersions of type II.

Let $p<m$ and consider $M_{o}^{4 p-1}$ a distribution of $M^{4 m+2}$ spanned by the vector field $\xi_{o}$ as defined by (3.5). Obviously, the manifold $M^{4 m+2} / M_{o}^{4 p-1}$ is an almost contact metric manifold with 3-structure. So, the canonical projection $f: M^{4 m+2} \rightarrow M^{4 m+2} / M_{o}^{4 p-1}$ is an almost Hermitian almost contact metric submersion of type II.

4.3. Submersions of type III. Let $\tilde{M}=M^{4 m} \times M^{\prime 4 m^{\prime}+2}$ be the Cartesian product of an almost quaternionic manifold with an almost Hermitian almost contact metric manifold as constructed in Section 4.1.

It is clear that $\tilde{M}$ is an almost Hermitian almost contact metric manifold. So, the canonical projection map $f: M^{4 m} \times M^{\prime 4 m^{\prime}+2} \rightarrow M^{\prime 4 m^{\prime}+2}$ is an almost Hermitian almost contact metric submersion of type III.

Indeed, setting $\tilde{\varphi}_{1}\left(D, D^{\prime}\right)=\left(J_{2} D, \varphi_{1}^{\prime} D^{\prime}\right), \tilde{\varphi}_{2}\left(D, D^{\prime}\right)=\left(J_{3} D, \varphi_{2}^{\prime} D^{\prime}\right)$, and $\tilde{J}\left(D, D^{\prime}\right)=$ $\left(J_{1} D, J^{\prime} D^{\prime}\right)$, we have $f_{*} \tilde{\varphi}_{1}\left(D, D^{\prime}\right)=f_{*}\left(J_{2} D, \varphi_{1}^{\prime} D^{\prime}\right)=\varphi_{1}^{\prime} D^{\prime}=\varphi_{1}^{\prime} f_{*}\left(D, D^{\prime}\right)$ which shows that $f_{*} \tilde{\varphi}_{1}=\varphi_{1}^{\prime} f_{*}$. In the same manner, we can show that $f_{*} \tilde{\varphi}_{2}=\varphi_{2}^{\prime} f_{*}$ and $f_{*} \tilde{J}=J^{\prime} f_{*}$.

ACKNOWLEDGMENT. I would like to express my heartfelt thanks to Prof. Bill Watson for his comments and assistance in finalizing this paper.

\section{REFERENCES}

[1] D. Chinea, Transference of structures on almost complex contact metric submersions, Houston J. Math. 14 (1988), no. 1, 9-22.

[2] A. Gray and L. M. Hervella, The sixteen classes of almost Hermitian manifolds and their linear invariants, Ann. Mat. Pura Appl. (4) 123 (1980), 35-58.

[3] Y.-Y. Kuo, On almost contact 3-structure, Tôhoku Math. J. (2) 22 (1970), 325-332.

[4] B. O'Neill, The fundamental equations of a submersion, Michigan Math. J. 13 (1966), 459469.

[5] S. Sasaki and Y. Hatakeyama, On differentiable manifolds with certain structures which are closely related to almost contact structure. II, Tôhoku Math. J. (2) 13 (1961), 281-294.

[6] T. Tshikuna-Matamba, Quelques classes des submersions métriques presque de contact [Some classes of almost contact metric submersions], Rev. Roumaine Math. Pures Appl. 35 (1990), no. 8-10, 705-721 (French).

[7] _ A classification of almost contact metric submersions based on the fibres, An. Ştiinț. Univ. Al. I. Cuza Iaşi Secț. I a Mat. 41 (1995), no. 1, 135-144.

[8] _ On the structure of the base space and the fibres of an almost contact metric submersion, Houston J. Math. 23 (1997), no. 2, 291-305.

[9]___ Submersions métriques presque de contact presque Hermitiennes de type II [Almost contact almost Hermitian metric submersions of type II], Problèmes Variationnels en Mathématiques Appliquées (Kinshasa, 1995), Ann. Fac. Sci. Univ. Kinshasa, Num. Spécial 3, Presses Univ. Kinshasa, Kinshasa, 1997, pp. 317-332 (French). 
[10] - Submersions Riemanniennes ne commutant pas avec les champs de tenseurs de structure [Riemannian submersions that do not commute with structure tensor fields], An. Ştiinţ. Univ. Al. I. Cuza Iaşi. Mat. (N.S.) 46 (2000), no. 2, 251-262 (French).

[11] B. Watson, Almost Hermitian submersions, J. Differential Geom. 11 (1976), no. 1, 147-165.

[12] _ Riemannian submersions and instantons, Math. Modelling 1 (1980), no. 4, 381-393.

[13] _ $, G, G^{\prime}$-Riemannian submersions and nonlinear gauge field equations of general relativity, Global Analysis-Analysis on Manifolds (T. M. Rassias, ed.), Teubner-Texte Math., vol. 57, Teubner, Leipzig, 1983, pp. 324-349.

[14] _ Almost contact metric 3-submersions, Int. J. Math. Math. Sci. 7 (1984), no. 4, 667688.

[15] _ The differential geometry of two types of almost contact metric submersions, The Mathematical Heritage of C. F. Gauss (G. M. Rassias, ed.), World Scientific Publishing, New Jersey, 1991, pp. 827-861.

T. Tshikuna-Matamba: Département de Mathématiques, Institut Supérieur Pédagogique, B.P. 282 - Kananga, Democratic Republic of the Congo

E-mail address: tshikmat@yahoo.fr 


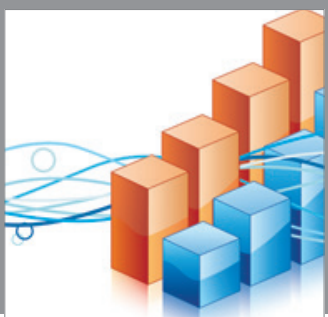

Advances in

Operations Research

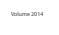

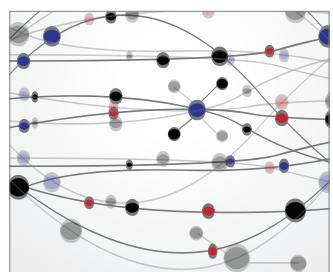

\section{The Scientific} World Journal
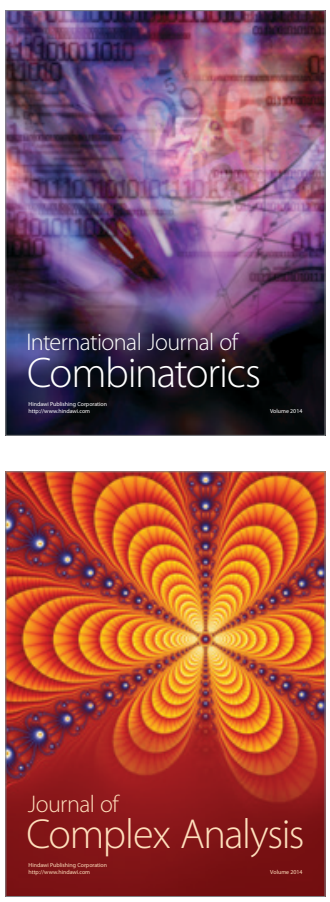

International Journal of

Mathematics and

Mathematical

Sciences
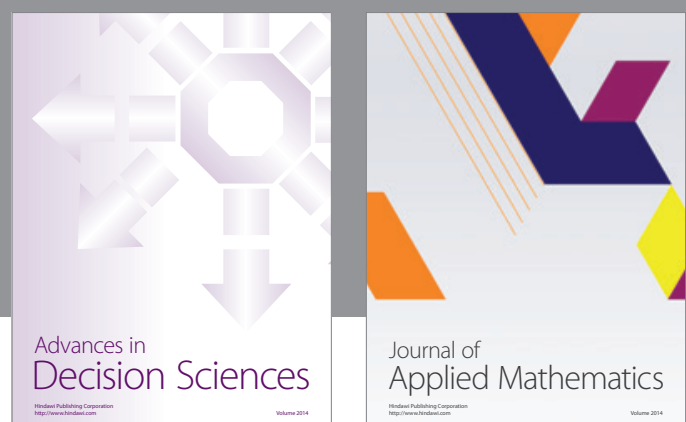

Journal of

Applied Mathematics
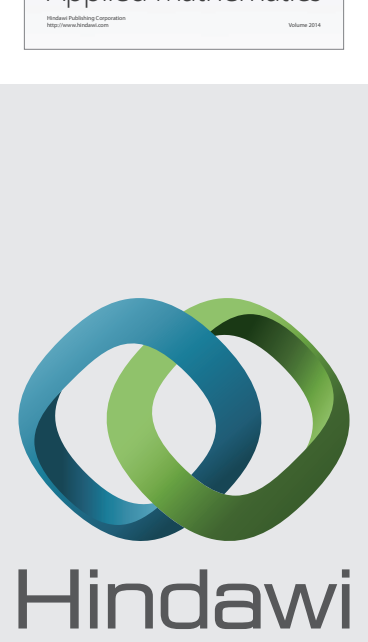

Submit your manuscripts at http://www.hindawi.com
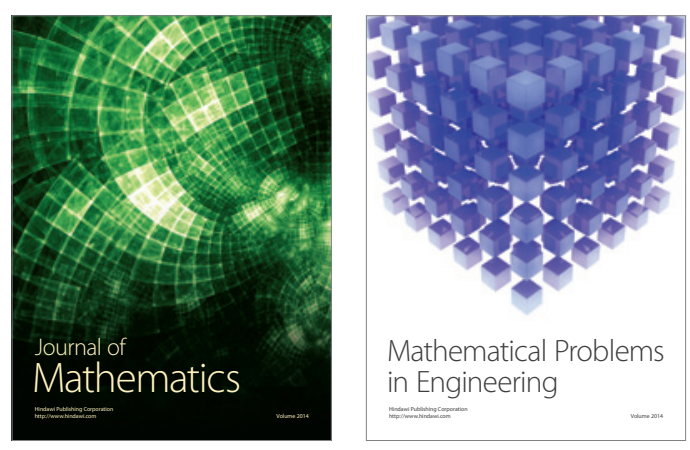

Mathematical Problems in Engineering
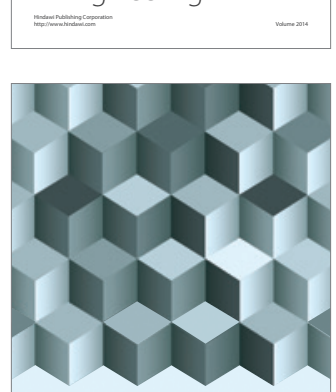

Journal of

Function Spaces
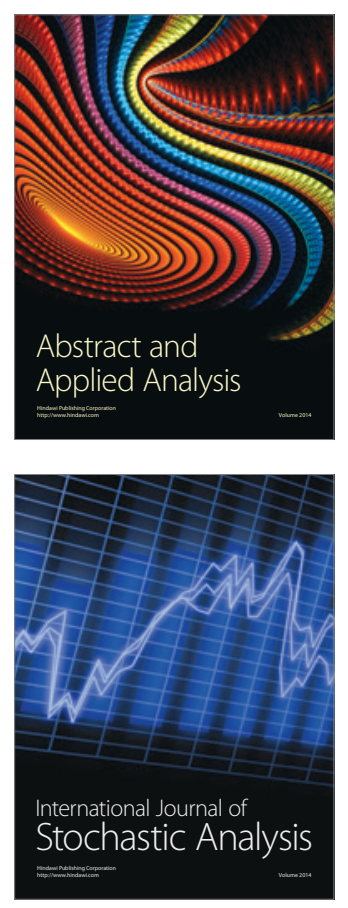

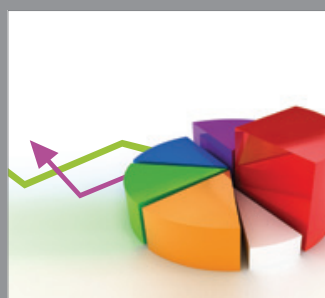

ournal of

Probability and Statistics

Promensencen
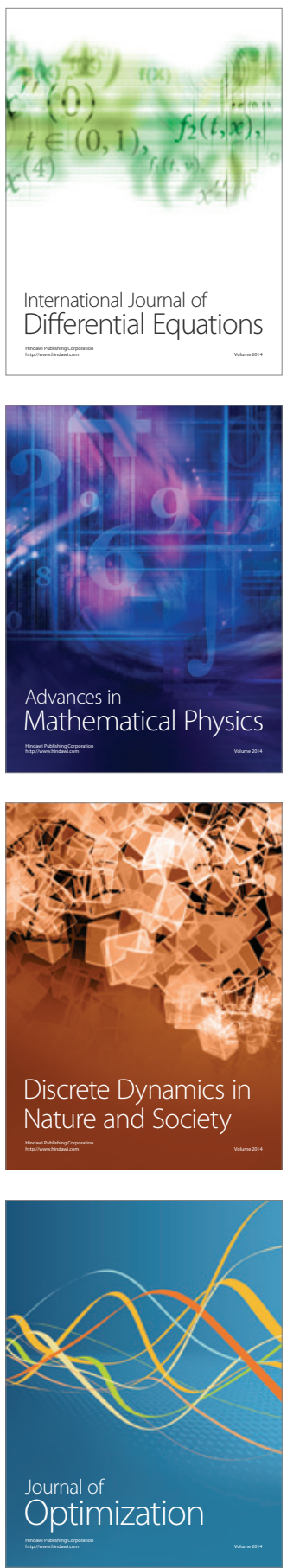